\title{
Layer-by-Layer Self-Assembled Ferrite Multilayer Nanofilms for Microwave Absorption
}

\author{
Jiwoong Heo, Daheui Choi, and Jinkee Hong \\ School of Chemical Engineering \& Materials Science, Chung-Ang University, Seoul 156-756, Republic of Korea \\ Correspondence should be addressed to Jinkee Hong; jkhong@cau.ac.kr
}

Received 30 August 2015; Revised 5 October 2015; Accepted 15 October 2015

Academic Editor: Miguel A. Correa-Duarte

Copyright (C) 2015 Jiwoong Heo et al. This is an open access article distributed under the Creative Commons Attribution License, which permits unrestricted use, distribution, and reproduction in any medium, provided the original work is properly cited.

\begin{abstract}
We demonstrate a simple method for fabricating multilayer thin films containing ferrite $\left(\mathrm{Co}_{0.5} \mathrm{Zn}_{0.5} \mathrm{Fe}_{2} \mathrm{O}_{4}\right)$ nanoparticles, using layer-by-layer (LbL) self-assembly. These films have microwave absorbing properties for possible radar absorbing and stealth applications. To demonstrate incorporation of inorganic ferrite nanoparticles into an electrostatic-interaction-based LbL selfassembly, we fabricated two types of films: (1) a blended three-component LbL film consisting of a sequential poly(acrylic acid)/oleic acid-ferrite blend layer and a poly(allylamine hydrochloride) layer and (2) a tetralayer LbL film consisting of sequential poly(diallyldimethylammonium chloride), poly(sodium-4-sulfonate), bPEI-ferrite, and poly(sodium-4-sulfonate) layers. We compared surface morphologies, thicknesses, and packing density of the two types of ferrite multilayer film. Ferrite nanoparticles $\left(\mathrm{Co}_{0.5} \mathrm{Zn}_{0.5} \mathrm{Fe}_{2} \mathrm{O}_{4}\right)$ were prepared via a coprecipitation method from an aqueous precursor solution. The structure and composition of the ferrite nanoparticles were characterized by X-ray diffraction, energy dispersive X-ray spectroscopy, transmission electron microscopy, and scanning electron microscopy. X-ray diffraction patterns of ferrite nanoparticles indicated a cubic spinel structure, and energy dispersive X-ray spectroscopy revealed their composition. Thickness growth and surface morphology were measured using a profilometer, atomic force microscope, and scanning electron microscope.
\end{abstract}

\section{Introduction}

Microwave absorbing materials, commonly known as radar absorbing materials, are in high demand for microwave absorption coatings on the surface of military stealth aircraft to avoid detection by enemy radar. A number of microwave absorbing materials have been explored for this application: conducting fibers $[1,2]$, ferromagnetic composites [3], carbon nanotubes [4], and ferrites [5-8] are frequently used. In particular, the unique microwave absorbing properties of ferrite nanoparticles, resulting from both magnetic and dielectric losses, have been explored by many groups [915]. Particularly, ferrite nanoparticles are widely used in the microwave absorbing technology. By controlling composition of ferrite, the performance of microwave absorbing can be tuned. Spinel ferrite has large resistivity and large magnetic loss; therefore, it has been used for microwave absorbing materials. Application of spinel ferrite is limited in $1-3 \mathrm{MHz}$ region because of its low resonance frequency; however, by doping with metallic ions such as $\mathrm{Co}, \mathrm{Zn}$, and $\mathrm{Mg}$ the absorbing range can be controlled which leads to unlimited possibility of spinel ferrite. For examples, Zhao group doped cobalt and cupper ions with $\mathrm{Ni}-\mathrm{Zn}$ spinel ferrite to tune the microwave absorbing properties. The microwave absorbing region, in which reflection loss values are lesser than $-10 \mathrm{bB}$, of $\mathrm{NiCoZnFe}_{2} \mathrm{O}_{4}$ is achieved at $3.9-11.5 \mathrm{GHz}$. The control of microwave absorption of metallic ion doped Ni-Zn ferrites mainly results from magnetic loss change derive from metallic ions [16].

At present, however, 3-dimensional arrangements of microwave absorbing materials are not well developed. Microwave absorbing materials are usually prepared as a suspension of colloidal nanoparticles in a solvent, in which electrostatic or steric repulsive forces prevent aggregation of nanoparticles [17], and conventional 3-dimensional arrays of magnetic nanoparticles are fabricated as pressed or mixed pellets [18-20]. However, these methods are costly and require an excessive volume of material, as the ferrite nanoparticles are not densely packed in the pellets. Therefore, a 
more effective method such as film deposition is needed for industrial applications.

Layer-by-layer (LbL) self-assembly consists of sequential adsorption of oppositely charged polymers to build up a multilayer thin film at the molecular level [21-24]. It allows the fabrication of multilayered thin films having desired thickness, composition, and density $[25,26]$. Moreover, various materials ranging from polyelectrolytes to nanoparticles [27], DNA [28], block copolymer micelles [29], and any kind of ferrite nanoparticles, such as cobalt ferrites [30], zin ferrites [31], and other mixed ferrites [32], can be incorporated into a LbLdeposited multilayer thin film through complementary interactions (e.g., electrostatic interactions, covalent bonding, hydrogen bonding, and hydrophobic interactions) [33-35].

There are previous studies about film deposition method that incorporates ferrite nanoparticles into multilayered film by LbL self-assembly [36-38].

In this study, we report a facile and simple method of preparing microwave absorbing nanofilm containing densely packed and well-organized ferrite nanoparticles. Since ferrite nanoparticles are inorganic, as-synthesized oleic-acid- (OA) stabilized nanoparticles do not disperse in water and are unsuitable for electrostatic interaction-based LbL self-assembly. Therefore, additional ligand exchange treatment is needed to incorporate ferrite nanoparticles in an LbL-assembled film. During this study, we examined two different types of films and compared their surface morphologies, thicknesses, and the packing density of incorporated ferrite nanoparticles. The blended three-component LbL film consists of a weak-polyelectrolyte/ferrite-nanoparticle-blended layer and an oppositely charged weak polyelectrolyte layer. Poly(allylamine hydrochloride) (PAH) and poly(acrylic acid) (PAA) are typically used weak polyelectrolytes for electrostatic interaction-based LbL self-assembly and are used as building blocks for fabricating blended three-component LbL films. Tetralayer LbL films consist of two kinds of strong polyelectrolytes and ferrite nanoparticles. Poly(diallyldimethylammonium chloride) (PDADMAC) and poly(sodium 4-styrenesulfonate) (PSS) are used as strong polyelectrolytes for tetralayer $\mathrm{LbL}$ films. The main difference between a blended three-component $\mathrm{LbL}$ film and a tetralayer $\mathrm{LbL}$ film is that the blended three-component LbL film can be built using as-synthesized oleic-acid- (OA-) stabilized ferrite nanoparticles without functionalization of the nanoparticles, whereas the tetralayer film needs ligand exchange of the ferrite nanoparticles. The effect of ligand-exchanged nanoparticles and the composition of two types of LbL selfassembled films are examined in this study.

\section{Material and Methods}

2.1. Materials. Iron(III) chloride hexahydrate, zinc sulfate heptahydrate, cobalt(II) chloride hexahydrate, oleic acid (OA), and sodium hydroxide powder were purchased from DaeJung. Trioctylphosphine oxide (TOPO), poly(diallyldimethylammonium chloride) solution (PDADMAC, $\mathrm{Mw}$ 200,000-350,000, 20 wt. \% in $\mathrm{H}_{2} \mathrm{O}$ ), poly(sodium 4-styrenesulfonate) (PSS, Mw 70,000), and branched polyethylenimine (bPEI, Mw 25,000) were obtained from Sigma Aldrich.

\subsection{Synthesis of Ferrite Nanoparticles}

2.2.1. Oleic-Acid- (OA-) Stabilized Ferrite Nanoparticles. Cobalt-doped zinc ferrite $\left(\mathrm{Co}_{0.5} \mathrm{Zn}_{0.5} \mathrm{Fe}_{2} \mathrm{O}_{4}\right)$ nanoparticles were prepared following the procedure reported by López et al. [39]. Briefly, $\mathrm{FeCl}_{3} \cdot 6 \mathrm{H}_{2} \mathrm{O}(15 \mathrm{~mL} ; 0.4 \mathrm{M}), \mathrm{CoCl}_{2} \cdot 6 \mathrm{H}_{2} \mathrm{O}$ $(15 \mathrm{~mL} ; 0.1 \mathrm{M})$, and $\mathrm{ZnSO}_{4} \cdot 7 \mathrm{H}_{2} \mathrm{O}(15 \mathrm{~mL} ; 0.1 \mathrm{M})$ solutions were mixed as the precursor solution. $\mathrm{NaOH}(15 \mathrm{~mL} ; 3 \mathrm{M})$ was added as the coprecipitation agent to the precursor solution, dropwise with vigorous stirring. Precipitation occurred immediately, changing the solution's color to brown. The precipitated solution was stirred at $80^{\circ} \mathrm{C}$ for $1 \mathrm{~h}$ for reaction. After reaction the $\mathrm{pH}$ of the solution changed from 12 to 10.5 by washing with DI water. To prevent aggregation of ferrite nanoparticles, $5 \mathrm{~mL}$ of $\mathrm{OA}$ was added to the solution as a coating agent and surfactant and then stirred for $2 \mathrm{~h}$ at $80^{\circ} \mathrm{C}$. The precipitate was washed twice with DI water and then washed with ethanol and acetone to remove unnecessary compounds. The supernatant was separated by centrifugation for $15 \mathrm{~min}$ at $4000 \mathrm{rpm}$, and the precipitate dried overnight in an $80^{\circ} \mathrm{C}$ oven.

2.2.2. bPEI Ligand-Exchanged Ferrite Nanoparticles. Trioctylphosphine oxide (TOPO) was added as cosurfactant when the oleic acid was introduced to the ferrite solution (TOPO: $1 \mathrm{~g}, 2.5 \mathrm{mmol}$; OA: $1 \mathrm{~mL}, 3.2 \mathrm{mmol})$. The remainder of the process was as above for the OA-stabilized ferrite nanoparticles. Well-dried OA + TOPO stabilized ferrite nanoparticles were dispersed into chloroform in a bath sonicator to obtain the ferrite solution $(5 \mathrm{mg} / \mathrm{mL})$. Ferrite solution $(2 \mathrm{~mL} ; 5 \mathrm{mg} / \mathrm{mL})$ was mixed with branched polyethylenimine (MW 25,000) in $100 \mathrm{~mL}$ of DMSO. After $48 \mathrm{~h}$ at room temperature for reaction, the bPEI-ferrite nanoparticles were collected by centrifugation for $1 \mathrm{~h}$ at $8000 \mathrm{rpm}$ and redispersed into $10 \mathrm{~mL}$ of DI water.

\subsection{Layer-by-Layer Assembly}

2.3.1. Blended Three-Component LbL Film. LbL multilayer films were fabricated by hand-dipping. The silicon wafer was exposed to $\mathrm{O}_{2}$ plasma to produce a negatively charged surface. The negatively charged wafer was dipped into cationic PAH solution ( $\mathrm{pH} 7.5$ ) for $15 \mathrm{~s}$ and then washed by dipping two times in DI water for $15 \mathrm{~s}$ to remove weakly attached polyelectrolytes. The PAA/ferrite blended layer, which follows in the sequence, was deposited on the PAH coated wafer surface by dipping the substrate into the blended solution of PAA and ferrite ( $\mathrm{pH}$ 3.5). Washing was repeated as in the previous step. The $\mathrm{pH}$ of the solution was adjusted using $\mathrm{NaOH}(0.2 \mathrm{M})$.

2.3.2. Tetralayer LbL Film. Following $\mathrm{O}_{2}$ plasma exposure, the negatively charged wafer was dipped into cationic PDADMAC solution for $10 \mathrm{~min}$ and washed three times by dipping in DI water for $2 \mathrm{~min}, 1 \mathrm{~min}$, and $1 \mathrm{~min}$ to remove weakly attached PDADMAC. The PSS, bPEI-ferrite, and PSS layers were then deposited in sequence on the PDADMAC layer, using the same adsorption process. The PDADMAC and PSS solutions contained $\mathrm{NaCl}(0.3 \mathrm{M})$. 


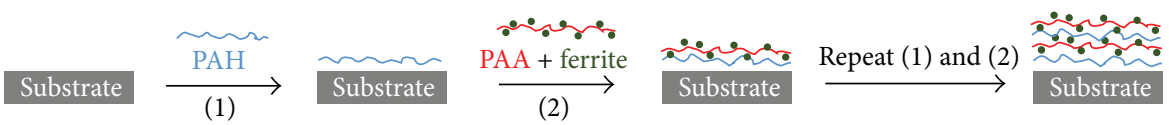

(a) Blended three-component LbL film

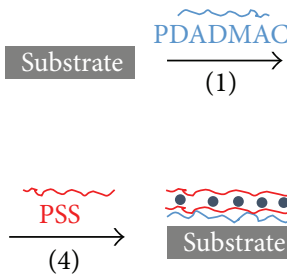

(4)

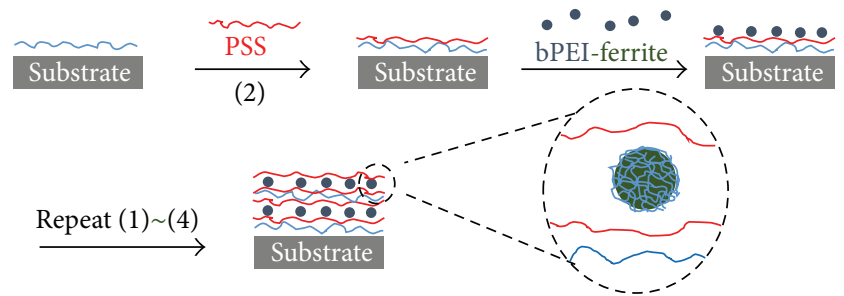

(b) Tetralayer LbL film

Scheme 1: Schematic of two types of layer-by-layer (LbL) nanofilm assembly on Si wafer. (a) Three-component blend LbL film (PAH/PAA + ferrite $)_{n}$, and (b) tetralayer LbL film (PDADMAC/PSS/bPEI-ferrite/PSS) ${ }_{n}$.

The processes for fabricating the two types of films are illustrated in Scheme 1.

\subsection{Characterization of Ferrite Nanoparticles and Multilayer} Films. Energy-filtering transmission electron microscopy (EF-TEM) images of ferrite nanoparticles were obtained using a LIBRA 120 microscope (Carl Zeiss). Energy-dispersive X-ray spectroscopy (EDX), in conjunction with fieldemission scanning electron microscopy (FE-SEM), was used for analysis of the dried ferrite nanoparticle powders. Atomic force microscopy (AFM) images were obtained with NX10 (Park Systems) in noncontact mode. The thickness of multilayer thin films on wafers was measured by Profilometer (Dektak 150, Veeco).

\section{Results and Discussion}

LbL self-assembly provides opportunities to control film thickness, composition, and morphology. Although intermolecular interactions such as hydrogen bonding, covalent bonding, and charge transfer can be adopted for LbL selfassembly, electrostatic interactions are most commonly used. During electrostatic interaction-based LbL assembly, the structure and morphology of fabricated multilayer thin film can be affected by parameters such as $\mathrm{pH}$, adsorption time, and concentration of solute and salt. The charge density of weak polyelectrolytes is tunable through its sensitivity to the solution's $\mathrm{pH}$. In comparison, the ionic strength of strong polyelectrolytes can be controlled through $\mathrm{NaCl}$ concentration. $\mathrm{NaCl}$ screens the repulsive interaction of strong polyelectrolytes, leading to an increased film thickness [40].

As-synthesized oleic-acid-stabilized ferrite nanoparticles do not disperse in water and require additional processes such as blending with charged polyelectrolytes or ligand exchange if they are to be used as building blocks for LbL-deposited films. Figure 1(a) presents a TEM image showing the size and morphology of OA-stabilized $\mathrm{Co}_{0.5} \mathrm{Zn}_{0.5} \mathrm{Fe}_{2} \mathrm{O}_{4}$ ferrite nanoparticles, while Figure 1(b) shows ligand-exchanged bPEI-ferrite nanoparticles.

The average size of synthesized OA-stabilized ferrite nanoparticles was about $18.5 \mathrm{~nm}$; in comparison, the ligandexchanged bPEI-ferrite nanoparticles average size was about
$7 \mathrm{~nm}$. The size reduction of ferrite nanoparticles originated in the added TOPO, which was introduced as a cosurfactant and coating agent to increase stability and to provide sufficient binding sites for bPEI ligands. This ligand exchange enabled water dispersion of inorganic nanoparticles: the amine group of the bPEI ligand protonated in the water solvent and had a positive zeta potential value of $+34.3 \mathrm{mV}$. The inset images of Figure 1 show ferrite nanoparticles in water: OA-stabilized ferrite nanoparticles did not disperse in water (Figure 1(a), left), while ligand-exchanged bPEI-ferrite nanoparticles were well dispersed (Figure 1(b), right). When magnetic nanoparticles are incorporated into film, a decreased magnetic dipole interaction between magnetic nanoparticles is important in creating a well-organized nanostructure.

$\mathrm{X}$-ray diffraction patterns of the powdered OA-stabilized ferrite nanoparticles confirmed a cubic spinel structure of $\mathrm{Co}_{0.5} \mathrm{Zn}_{0.5} \mathrm{Fe}_{2} \mathrm{O}_{4}$ (Figure 1(c)). Peaks indexed (220), (311), (400), (422), (511), and (440) were observed; a strong (311) plane reflection indicates the ferrite cubic spinel structure. The synthesized ferrite nanoparticles were observed to have good crystallinity and high quality, as there were no peaks observed from other phases. Microwave energy can be absorbed and transformed into heat energy by dielectric and magnetic losses when the absorber's characteristic impedance is matched to the characteristic impedance of free space [41]. Over the gigahertz $(\mathrm{GHz})$ range, dielectric losses are negligible $\left(\varepsilon^{\prime \prime} / \varepsilon^{\prime} \approx 0\right)$ and only magnetic loss affects microwave absorption. Spinel ferrites are familiar electromagnetic wave absorbers because of their resistivity and magnetic losses; however, at gigahertz frequencies, their permeability decreases according to Snoek's limit [42]. To increase microwave absorption properties, metal ions such as cobalt and zinc can be doped into spinel-type ferrites. Doped metallic ions change the permeability of ferrite nanoparticles which result in enhancement of microwave absorption properties by magnetic losses. The composition of synthesized ferrite nanoparticles was determined by EDX-SEM analysis (Figure 2 and Table 1), showing a molar ratio of about $0.5: 0.5: 2: 4[\mathrm{Co} / \mathrm{Zn} / \mathrm{Fe} / \mathrm{O}]$.

Because the $\mathrm{p} K_{a}$ value of PAA is 4.5 , the degree of ionization of PAA ( $\mathrm{pH} 3.5$ ) is about $30-40 \%$, which offers 


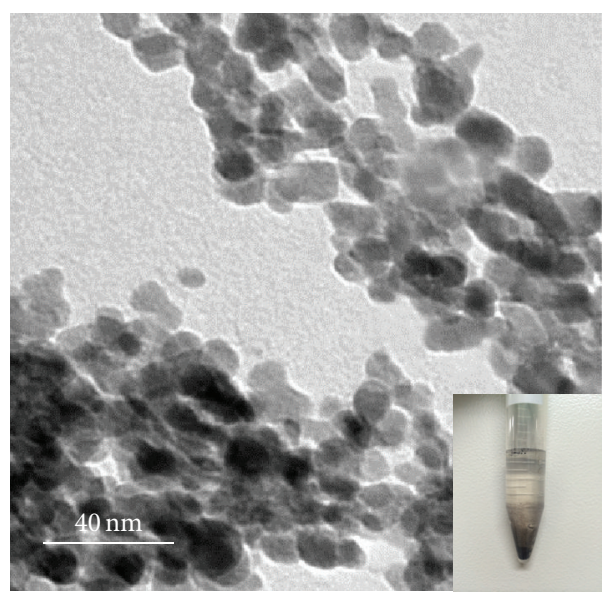

(a)

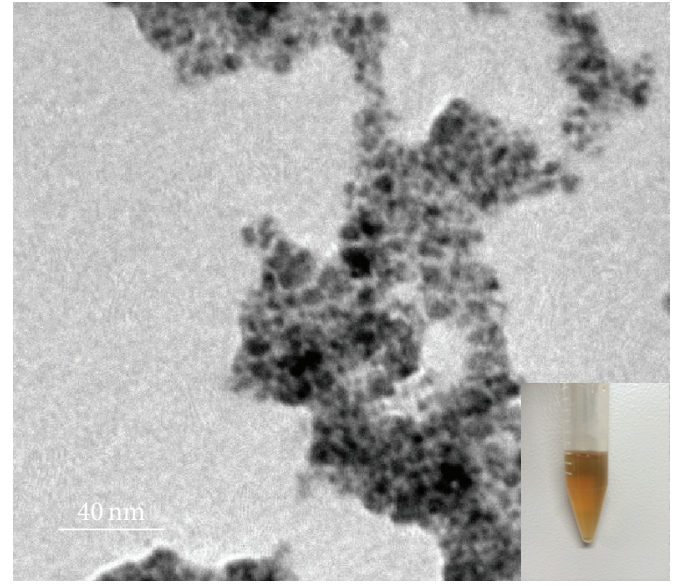

(b)

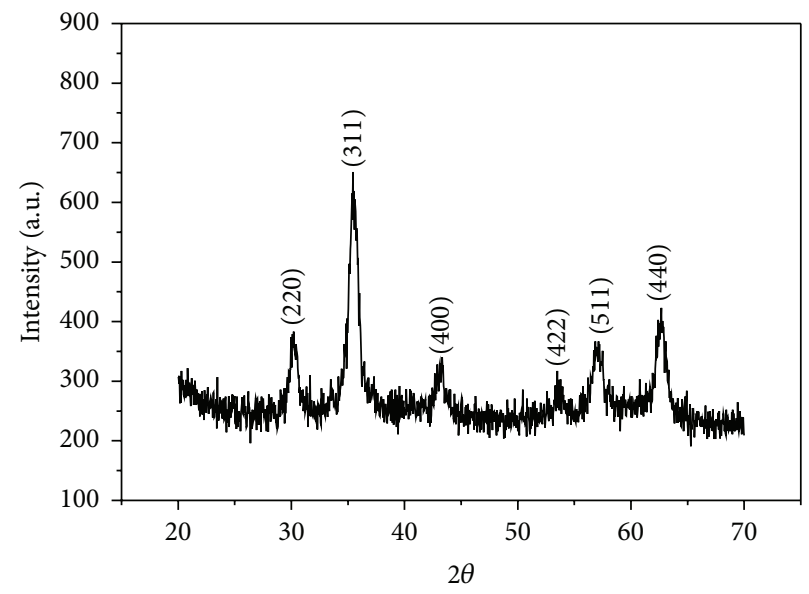

(c)

FIGURE 1: TEM images of (a) synthesized OA-stabilized $\mathrm{Co}_{0.5} \mathrm{Zn}_{0.5} \mathrm{Fe}_{2} \mathrm{O}_{4}$ ferrite nanoparticles, and (b) ligand-exchanged bPEI$\mathrm{Co}_{0.5} \mathrm{Zn}_{0.5} \mathrm{Fe}_{2} \mathrm{O}_{4}$ ferrite nanoparticles. Inset images show the ferrite nanoparticles from the respective treatments that (a) precipitated in water and (b) dispersed in water. (c) XRD patterns of powdered OA-stabilized $\mathrm{Co}_{0.5} \mathrm{Zn}_{0.5} \mathrm{Fe}_{2} \mathrm{O}_{4}$ nanoparticles.

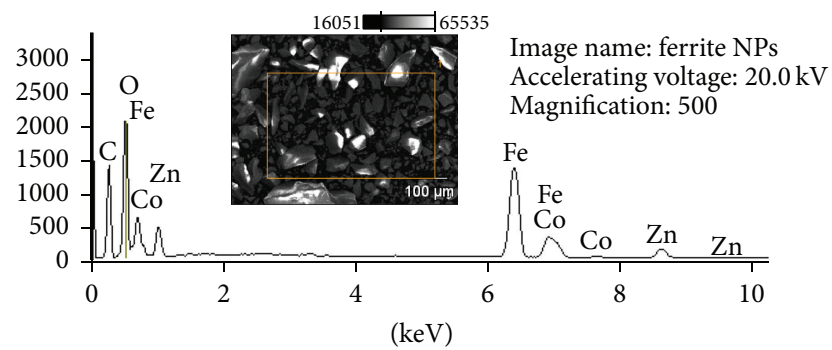

FIGURE 2: EDX spectrum of the as-prepared ferrite nanoparticles. Inset image shows the SEM top view of ferrite nanoparticles.

sufficient binding sites for blended ferrite nanoparticles and sequential PAH ( $\mathrm{pH}$ 7.5) layers [43]. Figure 3 shows the thickness growth curves of the two different types of LbL assembled film. The notation of LbL film listed as (PDADMAC/ PSS/bPEI-ferrite/PSS $)_{0.5 n}$ represents multilayer film consisting of PDADMAC, PSS, bPEI ligand-exchanged ferrite, and PSS where $n$ means the number of layers. For examples,

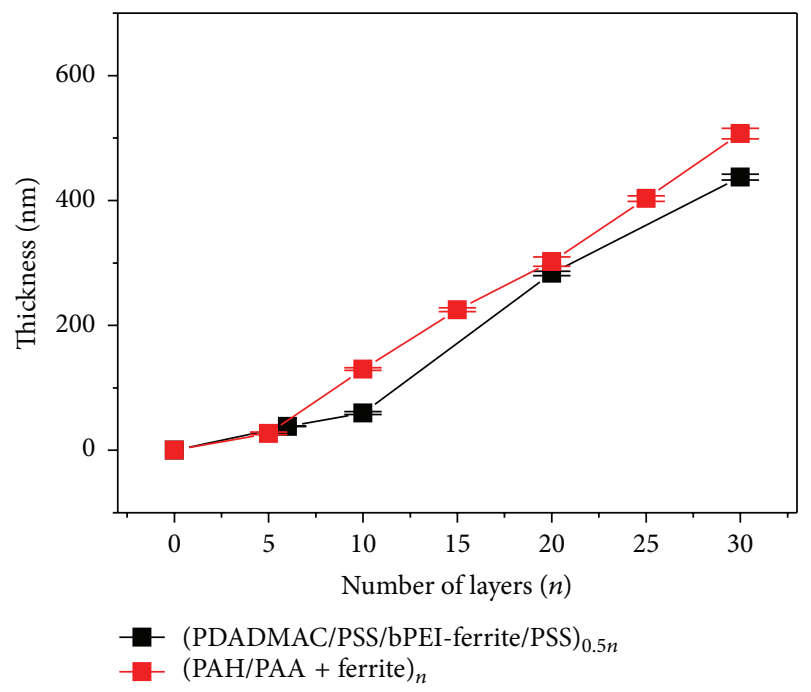

FIGURE 3: Growth curves of three-component blend LbL film (red line) and tetralayer LbL film (black line). 


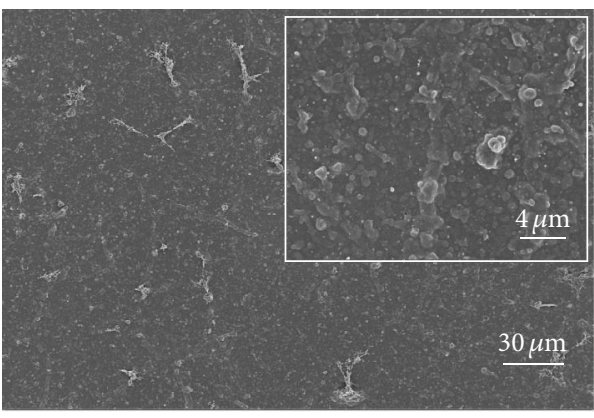

(a)

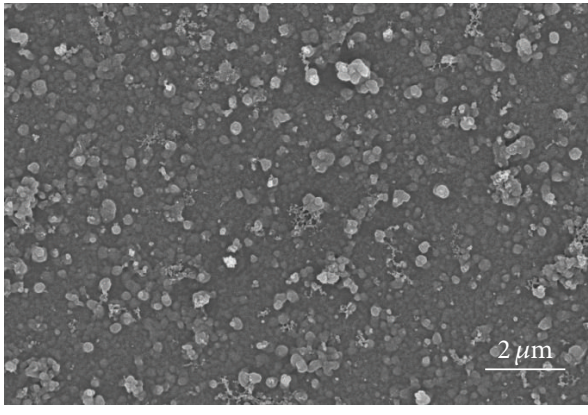

(b)

FIgURE 4: FE-SEM images of (a) blended three-component LbL film: (PAA + ferrite/PAH) ${ }_{10}$, and (b) tetralayer LbL film: (PDADMAC/ $\mathrm{PSS} / \mathrm{bPEI}$-ferrite/PDADMAC) ${ }_{10}$. Inset image in (a) shows higher magnification.

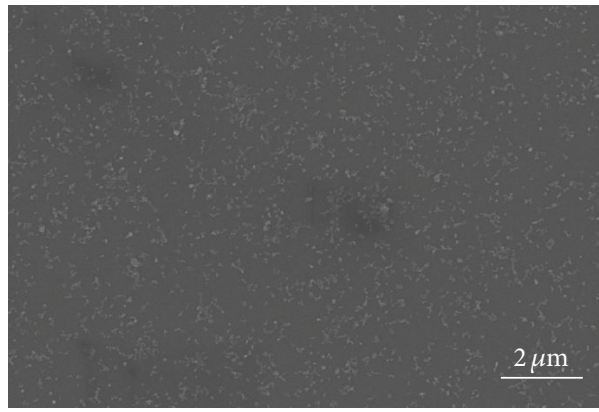

(a)

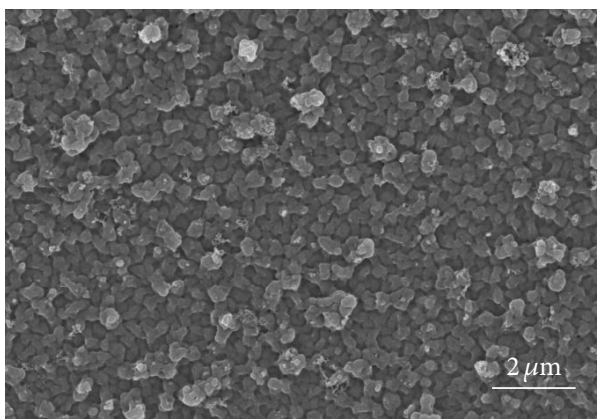

(c)

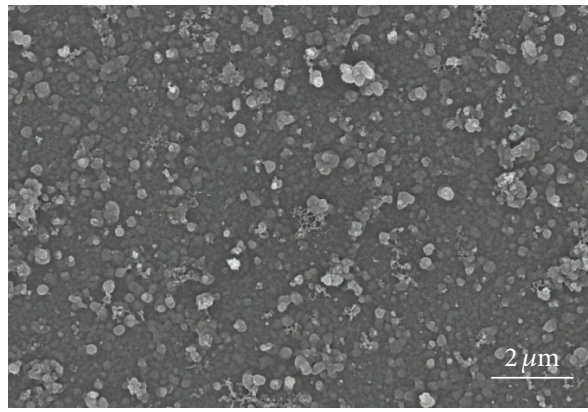

(b)

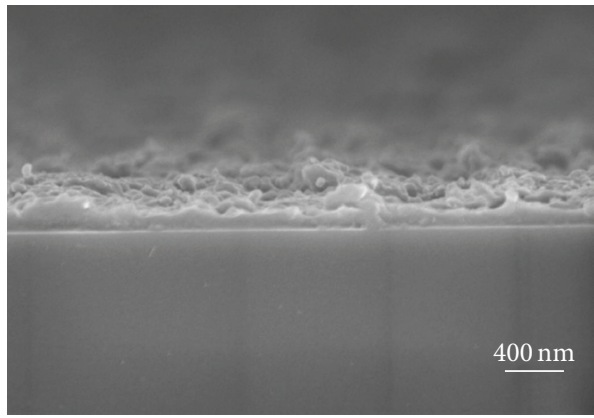

(d)

FIGURE 5: FE-SEM top-view images of tetralayer LbL films (PDADMAC/PSS/bPEI-ferrite/PSS) ${ }_{n}$. (a) $n=3$, (b) $n=10$, and (c) $n=15$. (d) A cross-sectional SEM image of (PDADMAC/PSS/bPEI-ferrite/PSS) $)_{10}$.

TABLE 1: Composition of the as-prepared ferrite nanoparticles.

\begin{tabular}{lcccc}
\hline Sample & Elements & Atomic \% & Weight \% & $\begin{array}{c}\text { Atomic ratio } \\
(\mathrm{Zn}=0.5)\end{array}$ \\
\hline & $\mathrm{Co}$ & 7.37 & 13.04 & 0.62 \\
$\mathrm{Co}_{0.5} \mathrm{Zn}_{0.5} \mathrm{Fe}_{2} \mathrm{O}_{4}$ & $\mathrm{Zn}$ & 5.87 & 11.53 & 0.50 \\
& $\mathrm{Fe}$ & 28.20 & 47.29 & 2.40 \\
& $\mathrm{O}$ & 58.56 & 28.14 & 4.98 \\
\hline
\end{tabular}

the notation of (PDADMAC/PSS/bPEI-ferrite/PSS) 10 where $n$ is 20 represents 10 -tetralayer multilayer film consisting of repeated PDADMAC/PSS/bPEI-ferrite and PSS. Thickness of the blended three-component LbL film (red line) increased linearly with the number of bilayers, indicating that the $\mathrm{PAA}+$ ferrite nanoparticle layer and the PAH layer successfully developed an LbL film. Thickness of the tetralayer LbL film also increased with the number of the bilayers, once five tetralayers had been deposited (black line). The top-view FESEM image shows the surface of the three-component LbL film consisting of (PAH/PAA + ferrite) ${ }_{10}$ (Figure 4(a)) and the tetralayer LbL film (PDADMAC/PSS/bPEI-ferrite/PSS) 10 (Figure 4(b)). The aggregation of ferrite nanoparticles in both films results from the nature of magnetic nanoparticles. Since ligand exchange of OA-stabilized ferrite nanoparticles with bPEI reduces the aggregation, the surface of the tetralayer LbL film shows much more densely packed ferrite nanoparticles than the blended three-component film. The key parameter 

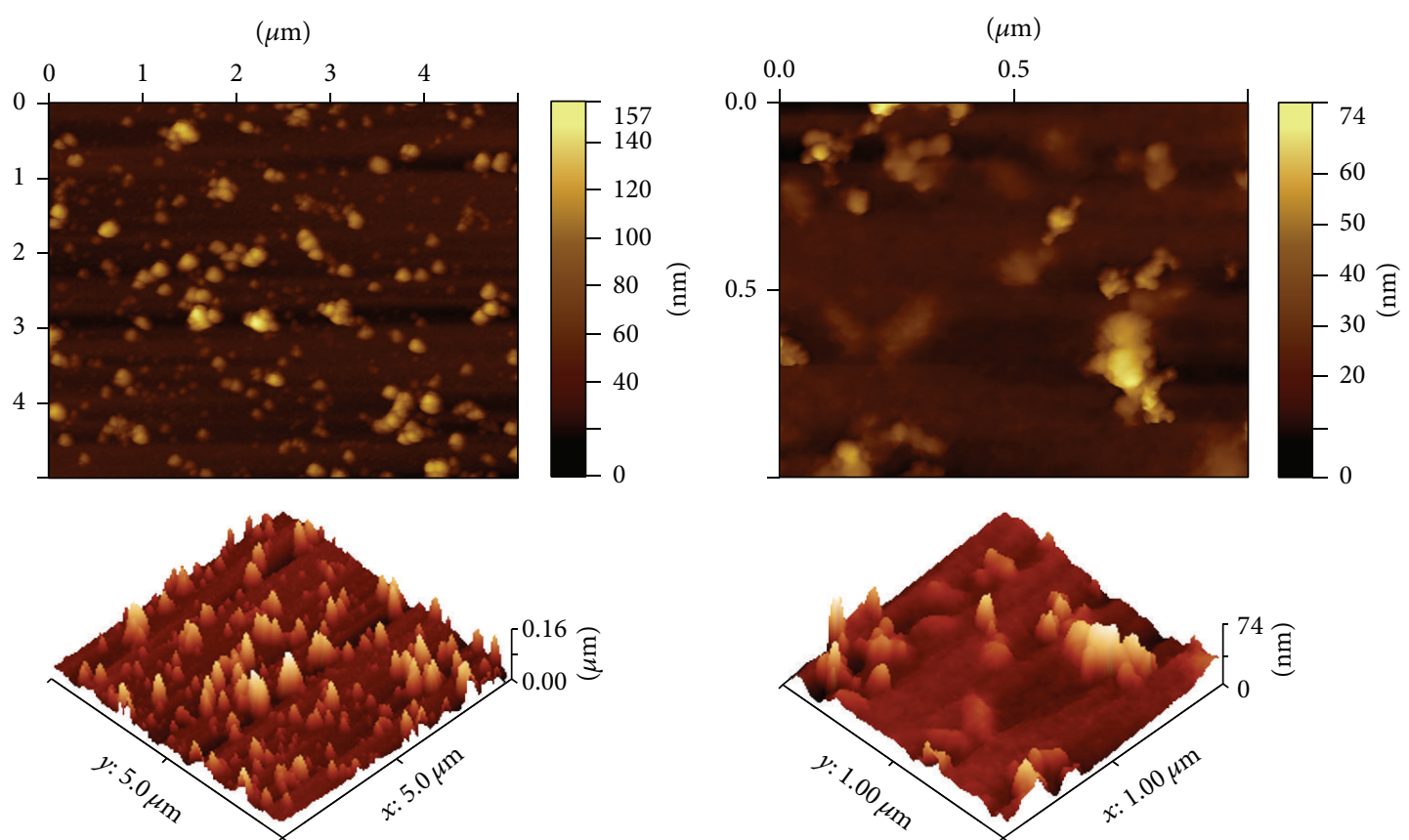

(a)

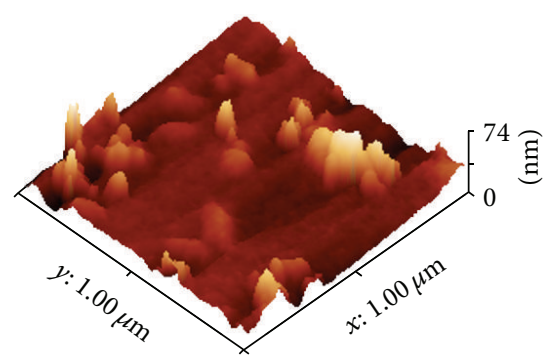

(b)
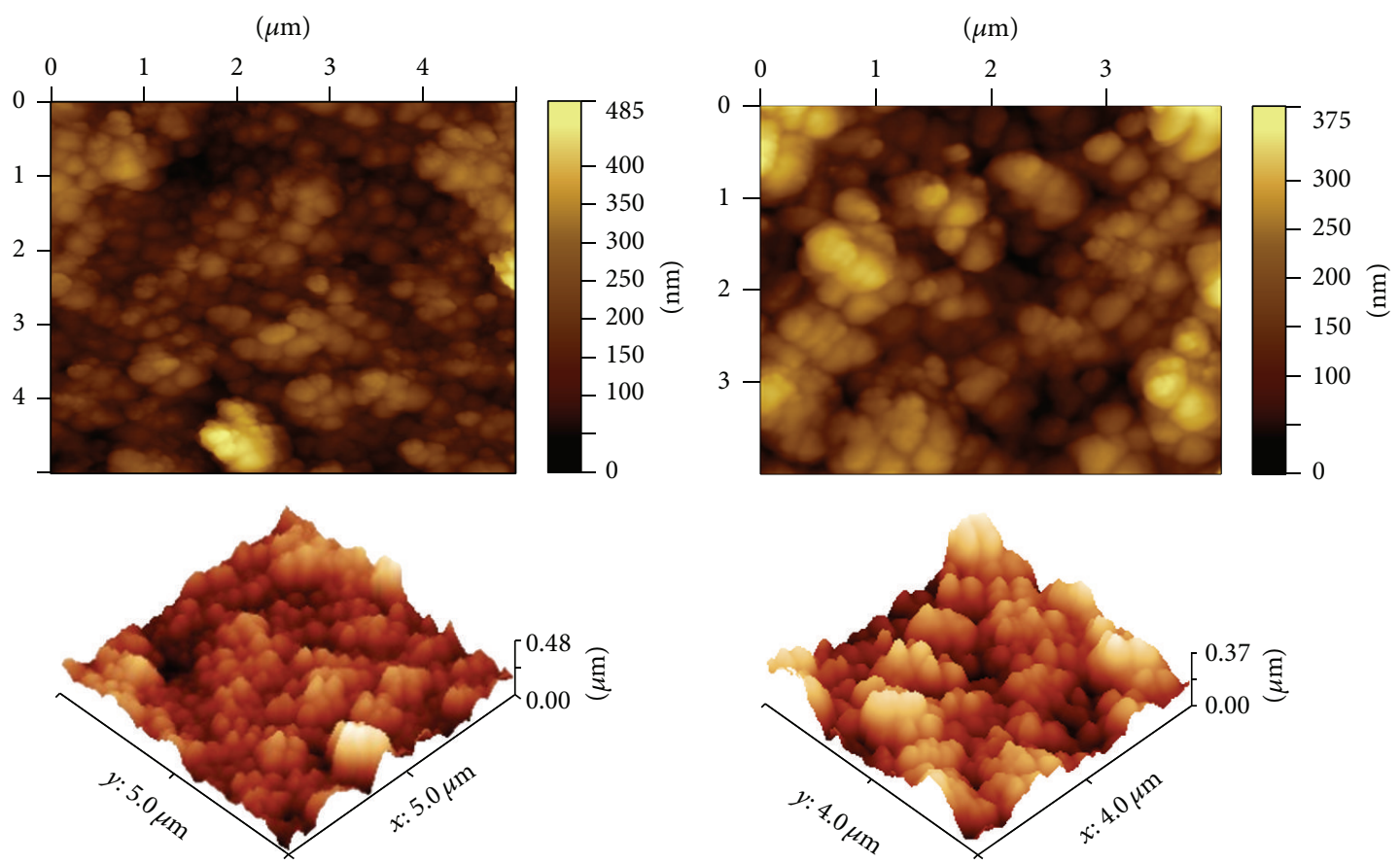

(c)

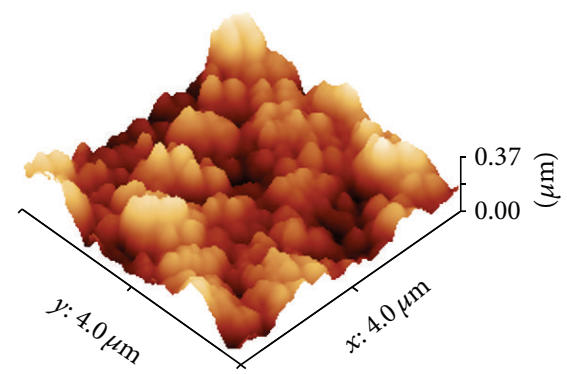

(d)

FIGURE 6: AFM images of tetralayer LbL film. (a) (PDADMAC/PSS/bPEI-ferrite/PDADMAC) 3 , (b) (PDADMAC/PSS/bPEI-ferrite/ PDADMAC $)_{5}$, (c) (PDADMAC/PSS/bPEI-ferrite/PDADMAC) ${ }_{10}$, and (d) (PDADMAC/PSS/bPEI-ferrite/PDADMAC) ${ }_{15}$.

for the phase distribution of ferrite nanoparticle is balance between attraction force of magnetic nanoparticles and repulsion force of positively charged bPEI ligand of ferrite particles [44]. Figures 5(a), 5(b), and 5(c) show top-view FE-SEM images of tetralayer LbL films. As the number of tetralayers increased, the surface density of ferrite nanoparticles gradually increased. The cross-sectional SEM image shows a homogeneously fabricated ferrite multilayer film that corresponds well to the thickness measured using a profilometer (Figure 3). The AFM height image (Figure 6) displays the surface morphology of tetralayer LbL film; ferrite nanoparticles did not fully cover the surface of the Si wafer until 10 tetralayers had been deposited. The root-meansquare $\left(R_{q}\right)$ surface roughness was $66.726 \mathrm{~nm}$ for 10 tetralayers and $67.052 \mathrm{~nm}$ for 15 tetralayers. These rough surfaces resulted from aggregation of ferrite nanoparticles caused by 
strong polyelectrolytes. Since the size of ligand-exchanged bPEI-ferrite nanoparticles was about $7 \mathrm{~nm}$ as measured from the TEM image (Figure 1(b)), the spherical structures appearing in Figure 6(c) indicate aggregates of tens of bPEIferrite nanoparticles. Even though the positively charged bPEI ligand counteracted the magnetic attraction between nanoparticles, an aggregated ferrite structure was observed.

\section{Conclusion}

In this study, we focused on incorporating ferrite nanoparticles into a multilayer nanofilm. The inorganic ferrite nanoparticles were oleic-acid- (OA-) stabilized or ligandexchanged using bPEI; the OA-stabilized nanoparticles had a diameter of about $18.5 \mathrm{~nm}$, compared to $7 \mathrm{~nm}$ after ligand exchange. We successfully fabricated two types of nanofilms: (1) a blended three-component LbL film consisting of PAA/ ferrite and $\mathrm{PAH}$ and (2) a tetralayer LbL film consisting of PDADMAC/PSS/bPEI-ferrite/PSS. In both methods, ferrite nanoparticles were deposited onto a $\mathrm{Si}$ wafer using an electrostatic-interaction-based LbL self-assembly method. Surface morphologies, thicknesses, and packing densities of the two types of film were investigated. Even though blended three-component LbL film is much simpler and faster than the tetralayer LbLfilm, the tetralayer LbL films had a better organized nanostructure of ferrite nanoparticles, in comparison to blended three-component LbL films. In conclusion, by employing LbL self-assembly, we can fabricate densely packed and well-organized multilayer ferrite films using either weak or strong polyelectrolytes. We believe that further research on this film deposition method for microwave absorbing ferrite nanoparticles will accelerate their industrial and military application in, for example, electronic devices, stealth aircraft, and other military technologies.

\section{Conflict of Interests}

The authors declare that there is no conflict of interests regarding the publication of this paper.

\section{Acknowledgments}

This research was supported by the Bio \& Medical Technology Development Program of the National Research Foundation of Korea (NRF), funded by the Korean government (no. 2012M3A9C6050104). Also, this work was supported by the National Research Foundation of Korea (NRF) grant funded by the Korea government Ministry of Science, ICT \& Future Planning (no. 2013R1A1A1076126). Additionally, this work was carried out with the support of "Cooperative Research Program for Agriculture Science \& Technology Development (Project no. PJ009986012015)" (Rural Development Administration, Republic of Korea), as well as the Korea Health Technology R\&D Project through the Korea Health Industry Development Institute (KHIDI), funded by the Ministry of Health \& Welfare (HI14C-3266-030014).

\section{References}

[1] M. Wu, H. He, Z. Zhao, and X. Yao, "Electromagnetic and microwave absorbing properties of iron fibre-epoxy resin composites," Journal of Physics D: Applied Physics, vol. 33, no. 19, pp. 2398-2401, 2000.

[2] W. Li, X. Qiao, H. Zhao, S. Wang, and Q. Ren, "Preparation and microwave absorbing properties of carbon/cobalt ferromagnetic composites," Journal of Nanoscience and Nanotechnology, vol. 13, no. 2, pp. 793-798, 2013.

[3] Y. Nie, H. He, Z. Zhao, R. Gong, and H. Yu, "Preparation, surface modification and microwave characterization of magnetic iron fibers," Journal of Magnetism and Magnetic Materials, vol. 306, no. 1, pp. 125-129, 2006.

[4] A. Wadhawan, D. Garrett, and J. M. Pérez, "Nanoparticleassisted microwave absorption by single-wall carbon nanotubes," Applied Physics Letters, vol. 83, no. 13, pp. 2683-2685, 2003.

[5] A. Yan, X. Liu, R. Yi, R. Shi, N. Zhang, and G. Qiu, "Selective synthesis and properties of monodisperse $\mathrm{Zn}$ ferrite hollow nanospheres and nanosheets," Journal of Physical Chemistry C, vol. 112, no. 23, pp. 8558-8563, 2008.

[6] A. Ghasemi, A. Saatchi, M. Salehi, A. Hossienpour, A. Morisako, and X. Liu, "Influence of matching thickness on the absorption properties of doped barium ferrites at microwave frequencies," Physica Status Solidi (A), vol. 203, no. 2, pp. 358-365, 2006.

[7] A. Ohlan, K. Singh, A. Chandra, and S. K. Dhawan, "Microwave absorption behavior of core-shell structured poly (3,4-ethylenedioxy thiophene)-barium ferrite nanocomposites," ACS Applied Materials and Interfaces, vol. 2, no. 3, pp. 927-933, 2010.

[8] W.-J. Wang, C.-G. Zang, and Q.-J. Jiao, "Fabrication and performance optimization of $\mathrm{Mn}-\mathrm{Zn}$ ferrite/EP composites as microwave absorbing materials," Chinese Physics B, vol. 22, no. 12, Article ID 128101, 2013.

[9] P. Singh, V. K. Babbar, A. Razdan, S. L. Srivastava, and T. C. Goel, "Microwave absorption studies of Ca-NiTi hexaferrite composites in X-band," Materials Science and Engineering B: Solid-State Materials for Advanced Technology, vol. 78, no. 2-3, pp. 70-74, 2000.

[10] S.-S. Kim and I.-K. Choi, "Design and fabrication of wideband ferrite absorber used in anechoic chamber," Journal of Magnetics, vol. 2, no. 1, pp. 25-27, 1997.

[11] A. M. Gama and M. C. Rezende, "Complex permeability and permittivity variation of radar absorbing materials based on mnzn ferrite in microwave frequencies," Materials Research, vol. 16, no. 5, pp. 997-1001, 2013.

[12] H. J. Kwon, J. Y. Shin, and J. H. Oh, “The microwave absorbing and resonance phenomena of Y-type hexagonal ferrite microwave absorbers," Journal of Applied Physics, vol. 75, no. 10, pp. 6109-6111, 1994.

[13] J. Y. Shin and J. H. Oh, "Microwave absorbing phenomena of ferrite microwave absorbers," IEEE Transactions on Magnetics, vol. 29, no. 6, pp. 3437-3439, 1993.

[14] P. Singh, V. K. Babbar, A. Razdan, S. L. Srivastava, and R. K. Puri, "Complex permeability and permittivity, and microwave absorption studies of $\mathrm{Ca}(\mathrm{CoTi})_{x} \mathrm{Fe}_{12-2 x} \mathrm{O}_{19}$ hexaferrite composites in X-band microwave frequencies," Materials Science and Engineering: B, vol. 67, no. 3, pp. 132-138, 1999.

[15] G.-M. Jeong, J. Choi, and S.-S. Kim, "Abnormal grain growth and magnetic loss in $\mathrm{Mn}-\mathrm{Zn}$ ferrites containing $\mathrm{CaO}$ and $\mathrm{SiO}_{2}$," IEEE Transactions on Magnetics, vol. 36, no. 5, pp. 3405-3407, 2000.

[16] D.-L. Zhao, Q. Lv, and Z.-M. Shen, "Fabrication and microwave absorbing properties of Ni-Zn spinel ferrites," Journal of Alloys and Compounds, vol. 480, no. 2, pp. 634-638, 2009. 
[17] D. Eberbeck, F. Wiekhorst, U. Steinhoff, and L. Trahms, “Aggregation behaviour of magnetic nanoparticle suspensions investigated by magnetorelaxometry," Journal of Physics: Condensed Matter, vol. 18, no. 38, pp. S2829-S2846, 2006.

[18] J. Jacob, M. A. Khadar, A. Lonappan, and K. T. Mathew, "Microwave dielectric properties of nanostructured nickel ferrite," Bulletin of Materials Science, vol. 31, no. 6, pp. 847-851, 2008.

[19] N. Gupta, M. C. Dimri, S. C. Kashyap, and D. C. Dube, "Processing and properties of cobalt-substituted lithium ferrite in the $\mathrm{GHz}$ frequency range," Ceramics International, vol. 31, no. 1, pp. 171-176, 2005.

[20] A. C. F. M. Costa, E. Tortella, M. R. Morelli, and R. H. G. A. Kiminami, "Synthesis, microstructure and magnetic properties of Ni-Zn ferrites," Journal of Magnetism and Magnetic Materials, vol. 256, no. 1-3, pp. 174-182, 2003.

[21] S. S. Shiratori and M. F. Rubner, "pH-dependent thickness behavior of sequentially adsorbed layers of weak polyelectrolytes," Macromolecules, vol. 33, no. 11, pp. 4213-4219, 2000.

[22] F. Caruso, R. A. Caruso, and H. Möhwald, "Nanoengineering of inorganic and hybrid hollow spheres by colloidal templating," Science, vol. 282, no. 5391, pp. 1111-1114, 1998.

[23] G. Decher, "Fuzzy nanoassemblies: toward layered polymeric multicomposites," Science, vol. 277, no. 5330, pp. 1232-1237, 1997.

[24] D. M. Lynn, "Peeling back the layers: controlled erosion and triggered disassembly of multilayered polyelectrolyte thin films," Advanced Materials, vol. 19, no. 23, pp. 4118-4130, 2007.

[25] K. C. Wood, J. Q. Boedicker, D. M. Lynn, and P. T. Hammond, "Tunable drug release from hydrolytically degradable layer-bylayer thin films," Langmuir, vol. 21, no. 4, pp. 1603-1609, 2005.

[26] J. Hong, B.-S. Kim, K. Char, and P. T. Hammond, "Inherent charge-shifting polyelectrolyte multilayer blends: a facile route for tunable protein release from surfaces," Biomacromolecules, vol. 12, no. 8, pp. 2975-2981, 2011.

[27] S. Srivastava and N. A. Kotov, "Composite Layer-by-Layer (LBL) assembly with inorganic nanoparticles and nanowires," Accounts of Chemical Research, vol. 41, no. 12, pp. 1831-1841, 2008.

[28] Y. Cho, J. B. Lee, and J. Hong, "Controlled release of an anti-cancer drug from DNA structured nano-films," Scientific Reports, vol. 4, no. 4078, pp. 1-4, 2014.

[29] J. Hong, Y. S. Kang, and S. W. Kang, "Nanoassembly of block copolymer micelle and graphene oxide to multilayer coatings," Industrial \& Engineering Chemistry Research, vol. 50, no. 6, pp. 3095-3099, 2011.

[30] A. M. Ibrahim, M. M. A. El-Latif, and M. M. Mahmoud, "Synthesis and characterization of nano-sized cobalt ferrite prepared via polyol method using conventional and microwave heating techniques," Journal of Alloys and Compounds, vol. 506, no. 1, pp. 201-204, 2010.

[31] A. N. Yusoff and M. H. Abdullah, "Microwave electromagnetic and absorption properties of some LiZn ferrites," Journal of Magnetism and Magnetic Materials, vol. 269, no. 2, pp. 271-280, 2004.

[32] S. Tyagi, H. B. Baskey, R. C. Agarwala, V. Agarwala, and T. C. Shami, "Development of hard/soft ferrite nanocomposite for enhanced microwave absorption," Ceramics International, vol. 37, no. 7, pp. 2631-2641, 2011.

[33] D. J. Schmidt and P. T. Hammond, "Electrochemically erasable hydrogen-bonded thin films," Chemical Communications, vol. 46, no. 39, pp. 7358-7360, 2010.
[34] J. P. DeRocher, P. Mao, J. Han, M. F. Rubner, and R. E. Cohen, "Layer-by-layer assembly of polyelectrolytes in nanofluidic devices," Macromolecules, vol. 43, no. 5, pp. 2430-2437, 2010.

[35] Z. Gemici, P. I. Schwachulla, E. H. Williamson, M. F. Rubner, and R. E. Cohen, "Targeted functionalization of nanoparticle thin films via capillary condensation," Nano Letters, vol. 9, no. 3, pp. 1064-1070, 2009.

[36] M. A. Correa-Duarte, M. Giersig, N. A. Kotov, and L. M. LizMarzán, "Control of packing order of self-assembled monolayers of magnetite nanoparticles with and without $\mathrm{SiO}_{2}$ coating by microwave irradiation," Langmuir, vol. 14, no. 22, pp. 64306435, 1998

[37] H. S. Kim, B. H. Sohn, W. Lee, J.-K. Lee, S. J. Choi, and S. J. Kwon, "Multifunctional layer-by-layer self-assembly of conducting polymers and magnetic nanoparticles," Thin Solid Films, vol. 419, no. 1-2, pp. 173-177, 2002.

[38] G. B. Alcantara, L. G. Paterno, F. J. Fonseca, P. C. Morais, and M. A. G. Soler, "Morphology of cobalt ferrite nanoparticlepolyelectrolyte multilayered nanocomposites," Journal of Magnetism and Magnetic Materials, vol. 323, no. 10, pp. 1372-1377, 2011.

[39] J. López, L. F. González-Bahamón, J. Prado et al., "Study of magnetic and structural properties of ferrofluids based on cobaltzinc ferrite nanoparticles," Journal of Magnetism and Magnetic Materials, vol. 324, no. 4, pp. 394-402, 2012.

[40] S. Ravati and B. D. Favis, "3D porous polymeric conductive material prepared using LbL deposition," Polymer, vol. 52, no. 3, pp. 718-731, 2011.

[41] Y. B. Feng, T. Qiu, and C. Y. Shen, "Absorbing properties and structural design of microwave absorbers based on carbonyl iron and barium ferrite," Journal of Magnetism and Magnetic Materials, vol. 318, no. 1-2, pp. 8-13, 2007.

[42] S. Zhu, K. Sun, Q. Y. Zhang, X. T. Zu, L. M. Wang, and R. C. Ewing, "Structural and magnetic characterization of $\mathrm{Co}_{x} \mathrm{Ni}_{1-x}$ nanoparticles in yttria-stabilized zirconia single crystals," Journal of Applied Physics, vol. 94, no. 9, pp. 5648-5651, 2003.

[43] S. Chibowski, M. Paszkiewicz, and J. Patkowski, "Adsorption of poly (acrylic acid) on the surface of $\mathrm{TiO}_{2}$ in the presence of different surfactants," Physicochemical Problems of Mineral Processing, vol. 48, no. 1, pp. 317-324, 2012.

[44] S. Laurent, D. Forge, M. Port et al., "Magnetic iron oxide nanoparticles: synthesis, stabilization, vectorization, physicochemical characterizations, and biological applications," Chemical Reviews, vol. 108, no. 6, pp. 2064-2110, 2008. 

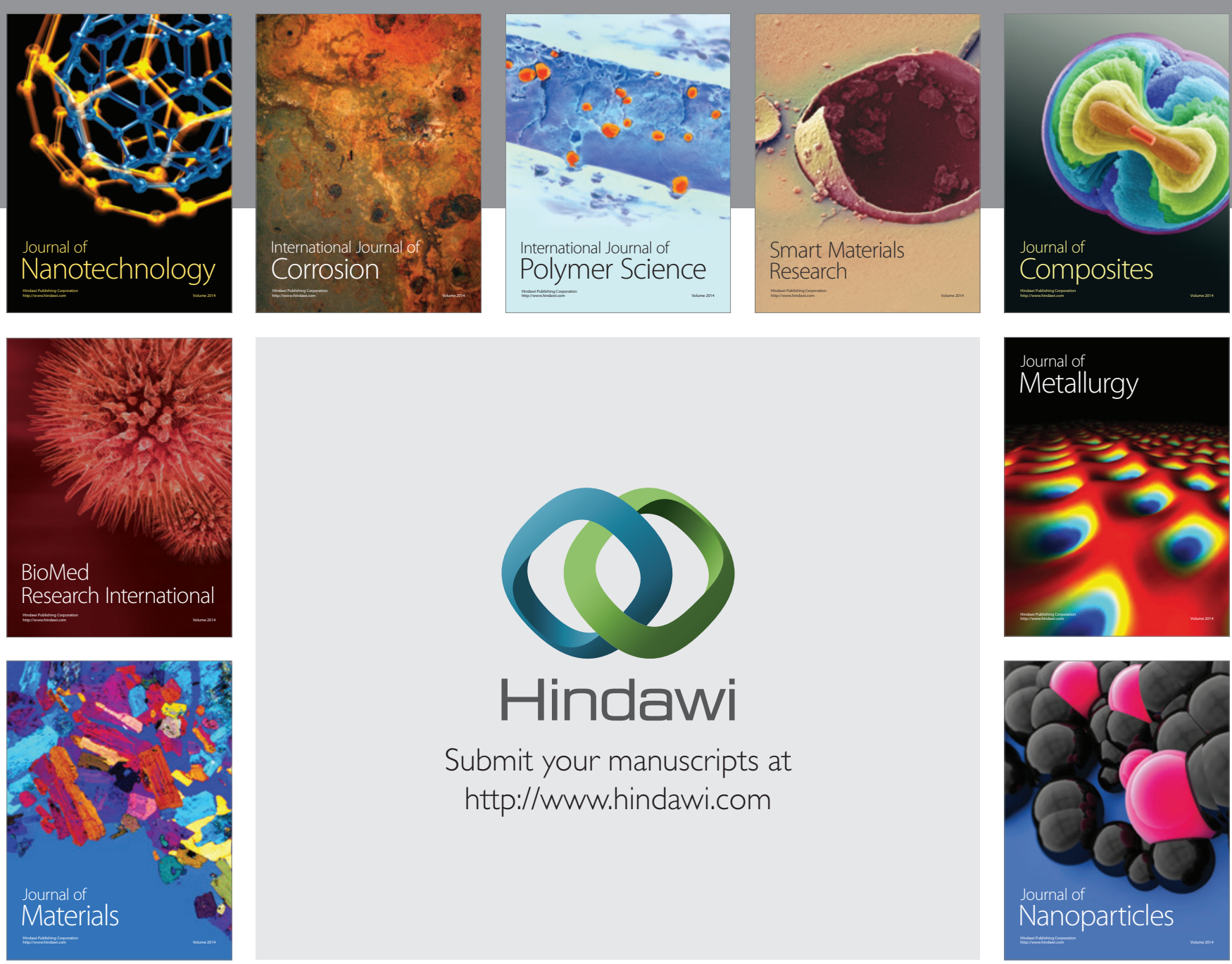

Submit your manuscripts at http://www.hindawi.com
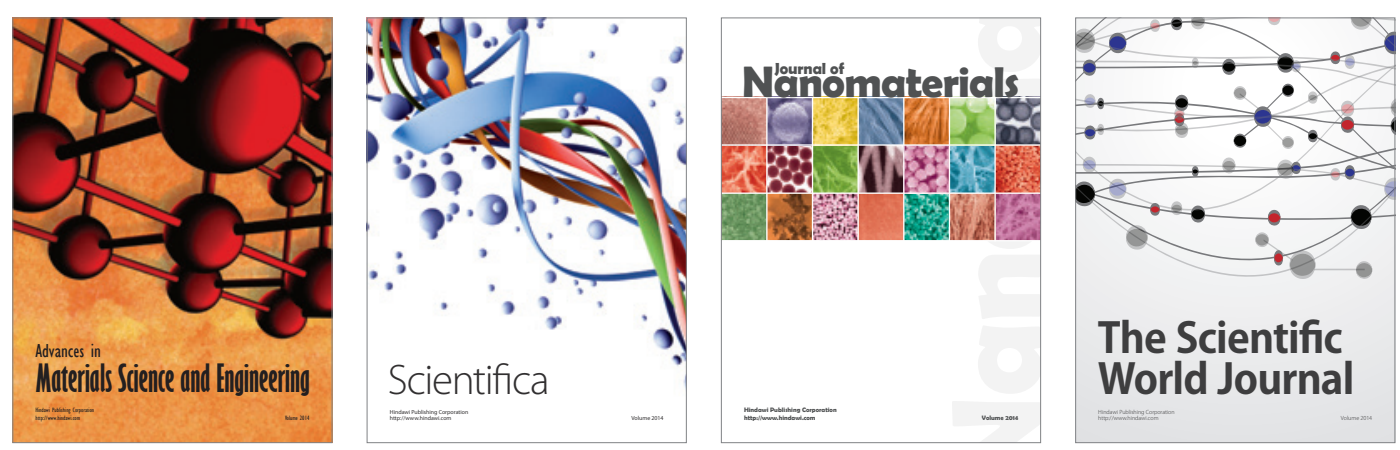

\section{The Scientific World Journal}
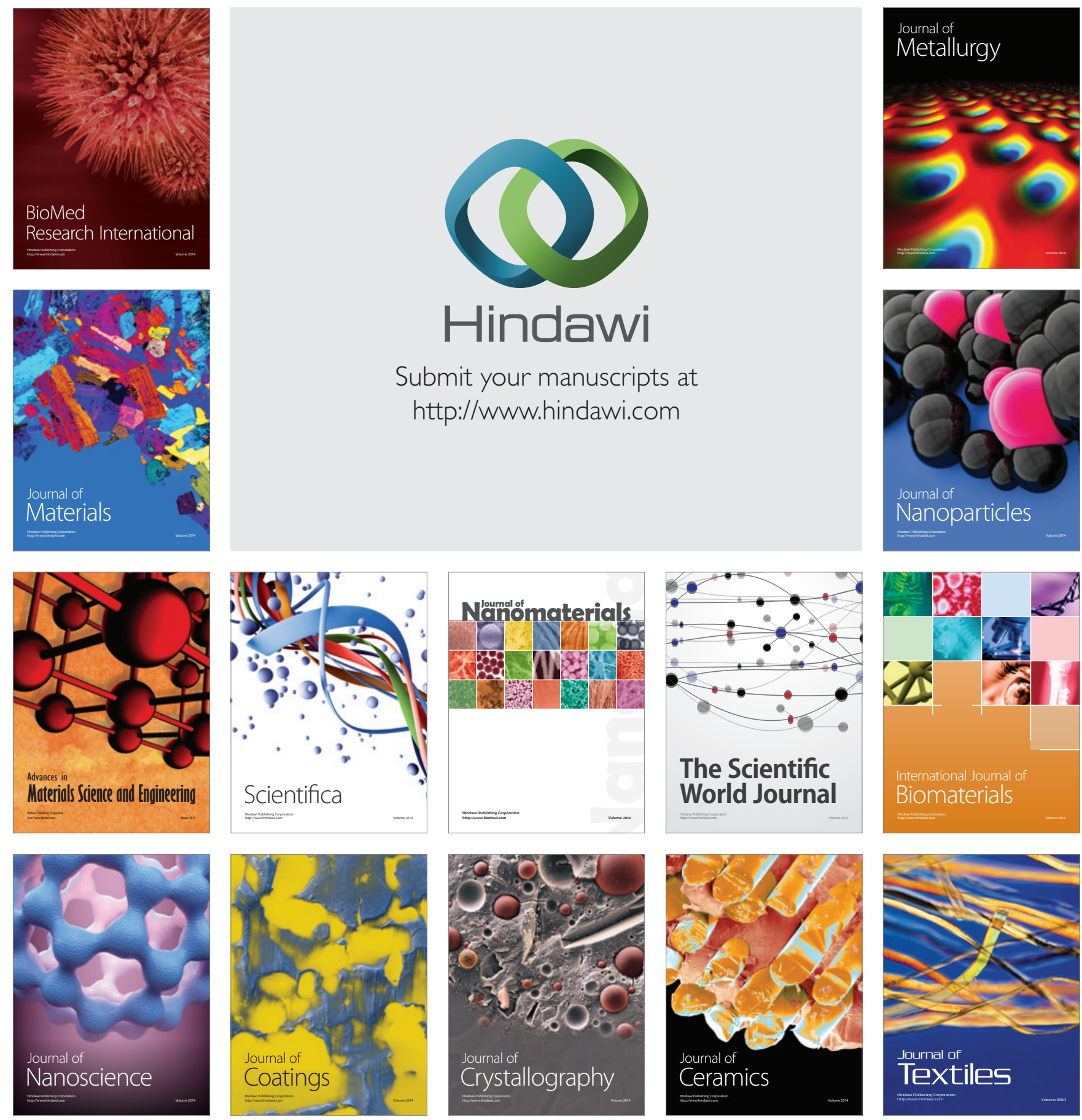